\section{Jugendliche Musikfans}

\section{STEFANIE RHEIN}

\section{Einleitung: Stereotype über Fans}

Insbesondere mit dem sogenannten TeenieFantum werden v. a. negative Bilder assoziiert:

- hysterisch kreischende Teenager

- jugendliche Einzelgänger, die versuchen, ihre Kontaktarmut durch eine intensive Fantasiebeziehung zu einem Star auszugleichen

- Jugendliche Konsumenten, denen die Person des Stars viel wichtiger ist als die Musik, und die unkritisch und beliebig vorfabrizierte Images anhimmeln.

Im Gegensatz zu diesen negativen Sichtweisen von Fantum, die teilweise auch in der wissenschaftlichen Behandlung des Themas vorzufinden sind, wird Fantum in der hier vorgestellten Arbeit als Beitrag zur jugendlichen Selbstsozialisation und Identitätskonstruktion aufgefasst. Fan-Sein wird verstanden als aktives (Medien-) Handeln, das sich im Kontext der Fangruppe bzw. Fangemeinde vollzieht.

Im Rahmen einer Befragung von 217 jugendlichen Fans und Nicht-Fans im Alter von 11 - 15 Jahren am MultiMediaComputer wurden $u$. a. die im Folgenden dargelegten Hypothesen untersucht.

\section{Theorie und Hypothesen}

Angenommen wurde, dass Teenie-Fantum die Mitgliedschaft in einer Jugendkultur bedeutet, die sich über ihren Musikgeschmack und durch spezifische Umgehensweisen mit populärmusikalischen Angeboten definiert. Über diese jugendkulturellen Stilmerkmale versichern sich die Fangruppen ihrer Zusammengehörigkeit sowohl nach innen als auch nach außen, sie grenzen sich dadurch darüber hinaus von anderen Personen und Gruppierungen ab (z. B. von Erwachsenen oder Nicht-Fans).

Über diese Verortung im sozialen bzw. kulturellen Raum wird Identität präsentiert. D afür ist es notwendig, dass sich der Fan bestimmte Wissensbestände (Experten-/ Fanwissen), Kompetenzen (z. B. die Tanzschritte aus dem Video von Britney Spears nachtanzen zu können) und Handlungsmuster (z. B. fan talk, Sammeln/ Tauschen von Fanartikeln) aneignet.

Die Aneignung vollzieht sich innerhalb der Gleichaltrigengruppe. Sie geht in der Regel autodidaktisch und kooperativ vonstatten und ist damit als Selbstsozialisation zu begreifen. Über das angeeignete Wissen und die relevanten Kompetenzen wird innerhalb der Fan- bzw. Gleichaltrigengruppe Anerkennung gewonnen.

Auf der Basis der skizzierten Überlegungen wird angenommen, dass sich Fans und Nicht-
Fans in ihren Umgehensweisen mit populärmusikalischen Angeboten unterscheiden. Unter anderem wurden folgende Hypothesen überprüft:

- Fans unterscheiden sich untereinander bezüglich der Bedeutung(en), die ihr Fan-Sein für sie hat. Davon wiederum hängt $a b$, wie stark sie sich in einzelnen Aspekten von Nicht-Fans unterscheiden.

- Fans und Nicht-Fans unterscheiden sich hinsichtlich der Kriterien, die ihnen wichtig sind, um Musik bzw. Musikstars oder gruppen besonders zu mögen..

- Fans nutzen ihren Musikgeschmack stärker als Nicht-Fans als Symbol der Zugehörigkeit und Abgrenzung und damit als Ausdruck kultureller Identität.

\section{Die Bedeutung von Fantum}

Die Unterscheidung zwischen Fans und NichtFans wurde über die Selbsteinschätzung der Befragten vorgenommen. $\mathrm{Zu}$ diesem Zweck wurde Fantum in einem Frageblock über die jugendkulturelle Orientierung durch zwei Items erhoben - Fans von M usikgruppen / -stars und Fans von Musikrichtungen. Jeder, der bei mindestens einem der beiden Items angibt, sich dazuzurechnen, wird als Teenie-Fan erfasst. 166 der Befragten sind damit zu den Fans zu zählen. D ies entspricht 76,5 \% der Stichprobe.

Um zwischen den Fans in der Stichprobe differenzieren zu können, wurden 12 verschiedene Bedeutungsaspekte von Fantum erhoben, zu denen die Befragten über eine 5-stufige Antwortskala Zustimmung oder Ablehnung ausdrücken konnten:

$\mathrm{E}$ in $\mathrm{F}$ an zu sein bedeutet fur mich,...

- ... die Musik gefunden zu haben, die mich begeistert."

- ... für einen Star zu schwärmen und von ihr/ ihm zu träumen."

- ... mich als Teil der Fangemeinschaft zu fühlen / eine A rt F amilie gefunden zu haben."

Aus den Ergebnissen lässt sich zum einen ablesen, dass bei den befragten Fans v. a. die musikbezogenen Aspekte im Mittelpunkt stehen, während die starbezogenen Bedeutungsitems der Schwärmerei bzw. des Bewunderns eine geringere Rolle spielen. Dieses Ergebnis widerspricht dem Stereotyp, dass die Person des Stars den Fans wichtiger sei als die Musik.

Geschlechtsspezifische Unterschiede in der Zustimmung $\mathrm{zu}$ den einzelnen Items ergeben sich nur bei zwei der zwölf Aspekte: Weibliche Fans stimmen sowohl dem Item Schwärmen als

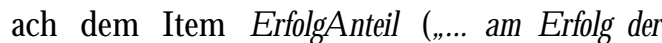
G ruppe/ des Stars A nteil zu haben.") signifikant stärker zu als die männlichen Fans. Bei beiden scheint es sich somit um eher weibliche Deutungsweisen von Fantum zu handeln. 
Über diese zwölf Items wurde eine Clusteranalyse durchgeführt. Entgegen der ursprünglichen Vermutung unterscheiden sich die gefundenen Fancluster gerade nicht in der Art der Bedeutung, die die Fans ihrem Fantum zuschreiben, sondern im Grad der Zustimmung zu den einzelnen Bedeutungsaspekten. D ie Cluster beschreiben folglich unterschiedliche Intensitäten von Fantum.

Auffällig ist, dass die Fans mit der niedrigsten Fan-Intensität (30 \% der Stichprobe) lediglich einem einzigen Item - auf relativ niedrigem $\mathrm{Ni}$ veau - zustimmen: E in Fan zu sein, bedeutet fur mich, die M usik gefunden zu haben, die mich begeistert. Diejenigen, die in diesem Cluster $\mathrm{zu}$ finden sind, werden als o.B.-Fans bezeichnet, wobei o.B. für „ohne Bedeutung” steht, da diese Fans ihren Fan-Sein offensichtlich kaum Bedeutung zuschreiben. D as Cluster mit der mittleren Intensität wird als „Normalfans” (31 \%), das mit der höchsten als „Superfans” (15\%) bezeichnet. Für die Superfans sind alle erhobenen Aspekte bedeutsam. deutung, die das Fan-Sein für die Jugendlichen hat, steigt folglich die Bedeutsamkeit, die sowohl den Auswahlkriterien insgesamt als auch den einzelnen Auswahlkriterien zugeschrieben wird.

Es kann auch hier gezeigt werden, dass die $\mathrm{Mu}$ sik für die Fans wichtiger ist als die Person des Stars: Als besonders wichtig werden - auch von den Superfans - die Aspekte M usik und Musikalisches Können betrachtet, dagegen befinden sich die starbezogenen Aspekte (A ussehen/ Outfit, Charakter/ V erhalten) auf den mittleren Plätzen. Während die Super- und die Normalfans die Musik an sich als „sehr wichtig” einstufen, wird diese von den Nicht-Fans sogar nur als „wichtig” betrachtet.

Anhand der Ergebnisse bei dem Kriterium, dass die Musik von den richtigen Leuten gehört wird, kann darüber hinaus gezeigt werden, dass mit der Intensität des Fantums auch die Wichtigkeit des Aspektes der sozialen Verortung durch den Musikgeschmack steigt. D ies wiederum verweist darauf, dass Fans ihren Musikge-

\section{Fan-Cluster}

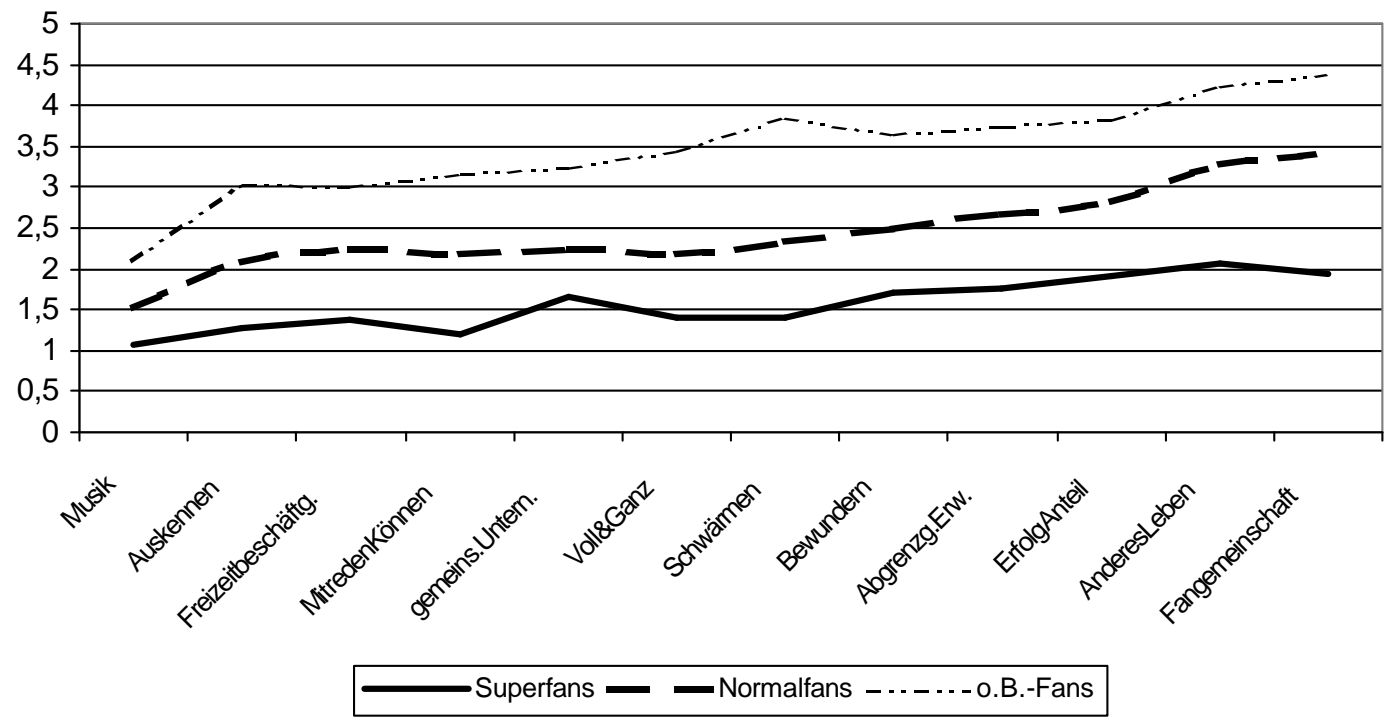

\begin{abstract}
Auswahlk riterien für die Lieblingsmusik
Erhoben wurde, wie wichtig Fans und NichtFans bestimmte Kriterien finden, damit ihnen ein Musikstück gefällt. Festgestellt werden kann, dass insgesamt alle erhobenen Kriterien als wichtig beurteilt wurden - unabhängig davon, ob es sich dabei um star-, musik- oder präsentationsbezogene Kriterien handelt. Jedoch finden die Fans in der Stichprobe jedes einzelne Kriterium signifikant wichtiger als die NichtFans, wobei die Superfans fast ausnahmslos immer die höchste Wichtigkeit zuschreiben und die Normalfans die zweithöchste. Mit der Be-
\end{abstract}

schmack stärker als Nicht-Fans zur Signalisierung von Nähe und Distanz und damit als Ausdruck ihrer kulturellen Identität sehen bzw. nutzen. 


\section{Kriterien für musik alische Präferenzen}

\begin{tabular}{|l|c|c|c|c|c|c|}
\hline $\begin{array}{l}\text { Kriterien } \\
\mathrm{n}=214\end{array}$ & Superfans & Nomalfans & o.B.-Fans & Nicht-Fans & Ges. & Sign. \\
\hline Musik an sich & 1,28 & 1,41 & 1,66 & 1,98 & 1,6 & $\mathrm{p}<0,001$ \\
\hline musikalisches Können & 1,88 & 1,59 & 1,97 & 2,10 & 1,86 & $\mathrm{p}<0,05$ \\
\hline Texte/ Botschaft & 1,81 & 2,13 & 2,52 & 2,38 & 2,26 & $\mathrm{p}<0,05$ \\
\hline Charakter/ Verhalten & 1,81 & 2,21 & 2,53 & 2,36 & 2,28 & $\mathrm{p}<0,05$ \\
\hline Musikvideos & 1,81 & 2,24 & 2,84 & 2,54 & 2,43 & $\mathrm{p}<0,001$ \\
\hline Bühnenshow & 2,03 & 2,41 & 2,88 & 2,82 & 2,59 & $\mathrm{p}<0,01$ \\
\hline Aussehen/ Outfit & 2,09 & 2,68 & 2,98 & 2,98 & 2,75 & $\mathrm{p}<0,01$ \\
\hline Richtige Leute & 2,31 & 2,78 & 3,56 & 2,82 & 2,95 & $\mathrm{p}<0,001$ \\
\hline
\end{tabular}

Wert 1: sehr wichtig; Wert 5: ganz unwichtig

\section{Zusammenfassung}

Fans schreiben ihrem Fan-Sein Bedeutungen in unterschiedlicher Intensität zu. Die subjektive Bedeutsamkeit der erhobenen Kriterien bei der Auswahl ihrer Lieblingsmusik steht in engem Zusammenhang mit der jeweiligen FanIntensität. Das Stereotyp, dass Fans den Star wichtiger finden als die Musik, trifft auf die befragten Fans nicht zu.

Desweiteren konnten Hinweise darauf gefunden werden, dass Fans ihren Musikgeschmack stärker als Nicht-Fans zur Verortung im sozialen Raum nutzen und damit als Ausdruck ihrer kulturellen Identität.

\section{Feedback}

\section{aus dem Protokoll von Timo D AMM}

Der Inhalt der Sitzung war sehr umfangreich und recht interessant. Es war jedoch schwierig, den Ausführungen $\mathrm{zu}$ folgen und alles $\mathrm{zu}$ notieren. Das lag aber zum größten Teil an dem Ausfall der Computeranlage. Viele Ausführungen wären wahrscheinlich auch anschaulicher gewesen.

Interessant fand ich die Ausführungen über die Talks sowie die Daily Soaps. Ich bin selber kein begeisterter "Soapschauer", kenne aber viele Leute, die sich diese Sendungen fast täglich ansehen und sich mit den Darstellern identifizieren. In meiner Schulzeit, als die Talks neu aufkamen, habe ich selbst ganze Nachmittage vor dem Fernseher verbracht und eine Talkshow nach der anderen angeschaut. In der Zwischenzeit schaue ich diese Sendungen schon lange nicht mehr.
Die Themen werden immer schlechter, das Interesse hat einfach nachgelassen aufgrund der Flut der Talks.

Viele Talkshows kommen mir fast gestellt vor. In den USA gibt es eine Talkshow (Jerry Springer), in der es regelmäßig zu Handgreiflichkeiten kommt.

Viele Schüler schauen Soaps und Talks, und somit finde ich es sinnvoll, etwas Hintergrundwissen darüber zu haben. 